\title{
COMPARACIÓN DE ESTRATEGIAS PARA LA ESTIMACIÓN DE ACTITUD EN CONTROLADORA DE VUELO PARA UAV PROFESIONAL
}

\author{
David Gallarta-Sáenz, Javier Rico-Azagra, Montserrat Gil-Martínez, Ramón Rico-Azagra \\ \{david.gallarta, javier.rico, montse.gil\}@unirioja.es, ramonrico92@gmail.com \\ Grupo de Investigación de Ingeniería de Control, Departamento de Ingeniería Eléctrica
}

Universidad de La Rioja

\section{Resumen}

Este trabajo presenta dos estrategias de integración multisensorial a través de las cuales se pretende encontrar un método apropiado para la estimación de actitud de un dron mediante filtros de Kalman y filtros complementarios explícitos. Para ello, se simulan los sensores de bajo coste, en concreto, unidades de medida inercial (IMU, Inertial Measurement Unit), que están incorporados en una controladora de vuelo diseñada para un UAV (Unmanned Aerial Vehicle) de ámbito profesional. De esta forma se dispone de unos datos fehacientes que resultan de gran interés para abordar la puesta en funcionamiento del sistema en pruebas reales, al poder implementar su sistema de control de forma adecuada en base a dicha información. La aplicación se desarrolla en MATLABSimulink@ por tratarse de un entorno adecuado para el trabajo con sistemas dinámicos.

Palabras clave: Estimación de Actitud, Unidad de Medida Inercial, Filtro Complementario Explícito, Filtro de Kalman, Controladora de Vuelo, UAV Profesional.

\section{INTRODUCCIÓN}

La estimación de la actitud $[18,19]$ es una tarea clave dentro del posicionamiento y la navegación de vehículos no tripulados $[4,17,20]$. Las técnicas de estimación han experimentado un importante crecimiento en los últimos años debido a la popularidad adquirida por este tipo de vehículos, así como por la evolución de los sistemas electromecánicos que han reducido tanto su tamaño como su precio de forma considerable, dando lugar a los denominados MEMS (Microelectromechanical Systems). Se trata de dispositivos accesibles y adecuados para embarcarlos en drones $[6,8]$ y en vehículos terrestres [1] sin que sus dimensiones supongan restricciones. Dentro de estos sistemas se encuentran los sensores de bajo coste, instrumentos encargados de adquirir los datos que posteriormente se procesarán para calcular información relativa al vehículo, como puede ser su actitud.
Por los motivos mencionados, las líneas de investigación en este campo se encuentra en pleno auge y están enfocadas, principalmente, en mejorar la precisión y en reducir el coste computacional de las pruebas, para conseguir sistemas eficientes teniendo en cuenta las limitaciones operativas de hardware asociadas a este tipo de vehículos.

El planteamiento de un simulador reduce el tiempo de ejecución de pruebas durante la primera fase de un proyecto, pudiendo conseguir gran cantidad de datos interesantes para la posterior ejecución del sistema de control, sin comprometer la integridad mecánica del dron. En este trabajo se modela el sistema de estimación incorporado en una controladora de vuelo de ámbito profesional, diseñada por el grupo de investigación Ingeniería de Control del Departamento de Ingeniería Eléctrica de la Universidad de La Rioja dentro del proyecto URpilot (Figura 1).

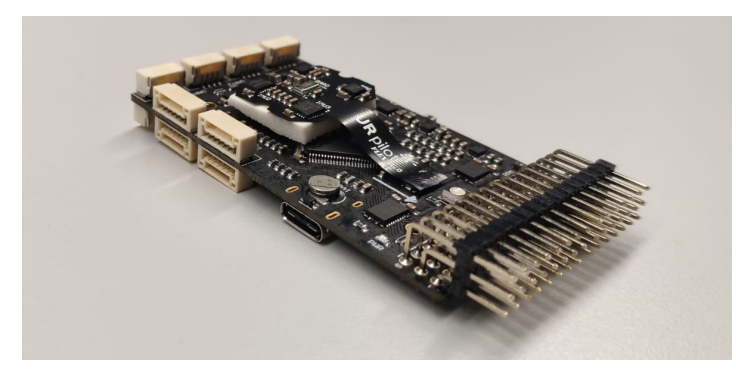

Figura 1: Controladora URpilot.

Para llevar a cabo esta aplicación también se modelan los sensores incorporados en dicha controladora, junto con los estimadores basados en filtros complementarios explícitos (ECF) [3, 15] y filtros de Kalman (KF) [11, 14]. De forma general, los filtros de Kalman consiguen estimaciones precisas gracias a su capacidad para mitigar el ruido del sistema, pero exigen un alto coste computacional. En la actualidad, estos algoritmos son empleados en múltiples aplicaciones: navegación [13], seguimiento de objetos [12] o fusión de datos de sensores $[5,10]$. Por su parte los filtros complementarios implican un menor coste computacional, por lo que son óptimos para dispositivos en los cuales la potencia de cálculo del microcontrolador es limitada. 
Para que el resultado de la estimación de la actitud en simulación sea lo más preciso posible, se proponen dos estrategias de fusión sensorial $[2,7]$ cuyas arquitecturas corresponden a integración filtrada en cascada de estado total e integración filtrada centralizada de estado total, respectivamente. En ellas se presentan diferentes combinaciones de los filtros mencionados para aprovechar al máximo sus virtudes, con el objetivo de simplificar el diseño de los mismos, es decir, el ajuste de los parámetros que los caracterizan, y de reducir el coste computacional. Asimismo, esta dualidad facilita la obtención de resultados de la misma naturaleza, al trabajar con diferentes modelos del mismo tipo de sensor, los cuales serán analizados y comparados para definir la técnica de estimación óptima para nuestro caso particular.

En el segundo apartado de este trabajo se explica el funcionamiento de la controladora de vuelo y el modelado de los sensores disponibles en ella. Posteriormente, se presentan las estrategias de estimación de actitud desarrolladas. Y, por último, los resultados alcanzados y las conclusiones extraídas.

\section{PLATAFORMA EMPLEADA}

\subsection{CONTROLADORA DE VUELO URpilot}

La controladora de vuelo desarrollada en el proyecto URpilot está compuesta por dos unidades funcionales (Figura 2). Por un lado, la unidad principal, denominada como «core», incorpora el microcontrolador STM32F767, los sistemas fundamentales y los conectores empleados para el conexionado de elementos externos. Esta unidad dispone de todos los sensores necesarios para el control de vuelo: una IMU ICM20689, un magnetómetro IST8310 y un barómetro MS5611. Además, lleva incorporado, como dispositivo adicional, un GPS que incluye un magnetómetro HMC5883.

Por otro lado, la unidad sensorial o «flex» integra sensores adicionales que mejoran la estimación del estado de la aeronave. Este segundo módulo incorpora tres IMUs (ICM20689, ICM20602 y MPU6000), un magnetómetro IST8310 y un barómetro MS5611. Como puede observarse en la Figura 1, la PCB se encuentra fabricada en material flexible y está concebida para situarse sobre la unidad principal. Este sistema permite aislar la unidad sensorial de las vibraciones producidas durante el vuelo, mejorando la adquisición de medidas por parte de los sensores.

En la Figura 3 se presenta un esquema modular de la controladora en el cual se detalla la electrónica embarcada y las conexiones existentes entre los distintos componentes.
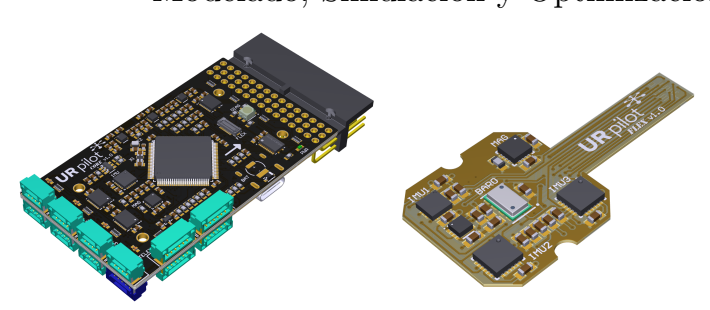

Figura 2: Unidad principal «core» (izq.) y unidad sensorial «flex»(dcha.).

El sistema de control de vuelo está ideado para que las estimaciones del estado del UAV realizadas durante el mismo se ejecuten empleando los sensores incorporados en la unidad sensorial, por ser los que se encuentra más aislados de las vibraciones que perjudican a la estimación. En caso de producirse un error en esta unidad, el sistema puede mantener la capacidad de vuelo gracias a los sensores incorporados en la unidad principal.

Durante el proceso de diseño de la controladora, para optimizar el funcionamiento de la misma, se decide programar los sensores en cuestión para muestrear a una frecuencia de $4 \mathrm{kHz}$, excepto el giroscopio perteneciente a MPU6000 que trabaja a $1 \mathrm{kHz}$.

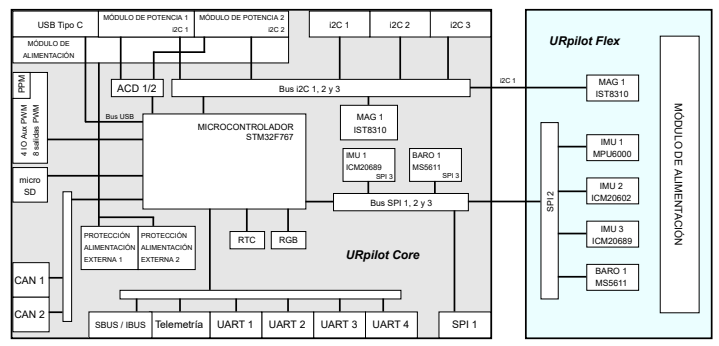

Figura 3: Esquema modular de la controladora de vuelo URpilot.

\subsection{SIMULADOR HARDWARE DE LOS SENSORES}

El modelado de los sensores se realiza mediante el bloque IMU simulation model disponible en la toolbox Sensor Fusion and Tracking de Simulink@, precisando sus parámetros a partir de la información proporcionada por el fabricante en las hojas de características.

Los datos de entrada necesarios en dicho bloque son la aceleración lineal, la velocidad angular y la orientación de referencia tomadas en el sistema de coordenadas de navegación local, obteniendo como datos de salida las medidas del acelerómetro, del giroscopio y del magnetómetro en el sistema de coordenadas del cuerpo. Esta es la información utilizada por los filtros para estimar la actitud.

En la tabla 1 se muestran las características más relevantes de ICM20689, ICM20602 y MPU6000. 
Tabla 1: Características principales de las IMUs.

\begin{tabular}{|c|c|c|c|}
\hline \multicolumn{2}{|l|}{ Sensor } & Bias & $\begin{array}{l}\text { Ruido } \\
\text { espectral }\end{array}$ \\
\hline ICM20689 & $\begin{array}{l}\text { Acel. } \\
\text { Giro. }\end{array}$ & $\begin{array}{ll} \pm 80 & \mathrm{mg} \\
\pm 5 & \circ / \mathrm{s}\end{array}$ & $\begin{array}{ll}150 & \mu \mathrm{g} / \sqrt{\mathrm{Hz}} \\
0.006 & \circ / \mathrm{s} / \sqrt{\mathrm{Hz}}\end{array}$ \\
\hline ICM20602 & & $\begin{array}{ll} \pm 25 & \mathrm{mg} \\
\pm 1 & \circ / \mathrm{s}\end{array}$ & $\begin{array}{ll}100 & \mu \mathrm{g} / \sqrt{\mathrm{Hz}} \\
0.004 & \circ / \mathrm{s} / \sqrt{\mathrm{Hz}}\end{array}$ \\
\hline & $\begin{array}{l}\text { Acel. } \\
\text { Giro. }\end{array}$ & $\begin{array}{ll}\mathrm{XY} \pm 50 \mathrm{mg} \\
\mathrm{Z} \pm 80 \mathrm{mg} \\
\pm 20 \quad \% / \mathrm{s}\end{array}$ & $\begin{array}{ll}400 & \mu \mathrm{g} / \sqrt{\mathrm{Hz}} \\
0.005 & \circ / \mathrm{s} / \sqrt{\mathrm{Hz}}\end{array}$ \\
\hline
\end{tabular}

Además, tal y como se indica en la hojas de características, estos sensores disponen de cuatro modos de funcionamiento que permiten ajustar su rango de escala completa (I) y su factor de escala de sensibilidad (II). Todos ellos se programan en el denominado modo 0 , definiendo los siguientes valores: $\pm 250 \%$ s (I) y $131 \mathrm{LSB} /(\% / \mathrm{s})$ (II) en lo que respecta al giroscopio y $\pm 2 \mathrm{~g}$ (I) y $16384 \mathrm{LSB} / \mathrm{g}$ (II) para el acelerómetro.

\section{ARQUITECTURAS DE INTEGRACIÓN MULTISENSORIAL}

En este apartado se explica la estructura y el funcionamiento de las estrategias de integración multisensorial planteadas para la estimación de actitud, cuyos resultados se analizan y comparan para elegir la solución que más se adecue a nuestro sistema.

En la práctica, el ajuste de los parámetros para los distintos filtros se realiza ad hoc en base a resultados experimentales, hasta conseguir el comportamiento deseado.

\subsection{ESTRATEGIA 1: INTEGRACIÓN FILTRADA EN CASCADA DE ESTADO TOTAL}

Esta arquitectura (Figura 4), en primer lugar, aplica un estimador independiente en los sensores. En concreto, se utiliza un ECF para procesar los datos de cada IMU, obteniendo múltiples valores de roll y pitch. De esta forma, la solución de cada uno de los subsistemas de navegación compone el vector de medidas $z$ del filtro de Kalman. De forma teórica, $z$ es el vector que contiene las medidas de los sensores, afectadas por ruido, y que actúan como observaciones en el filtro.

Posteriormente, mediante un filtro de Kalman se integran los valores de roll y pitch de las diferentes IMUs. Tras analizar sus características y los resultados previos, se opta por utilizar únicamente los datos de dos sensores, ICM20689 e ICM20602, ya que se espera, a priori, un mejor comportamiento.
La arquitectura actual no toma ningún subsistema como referencia, al contrario que ocurre en la integración filtrada en cascada de estado de error, y ante la previsible distorsión de los resultados se omite la información de MPU6000.

En definitiva, la solución integrada para este primer caso comprende los resultados provenientes de los ECF corregidos mediante estimaciones de su actitud realizadas por el algoritmo de integración, siendo este un filtro de Kalman.

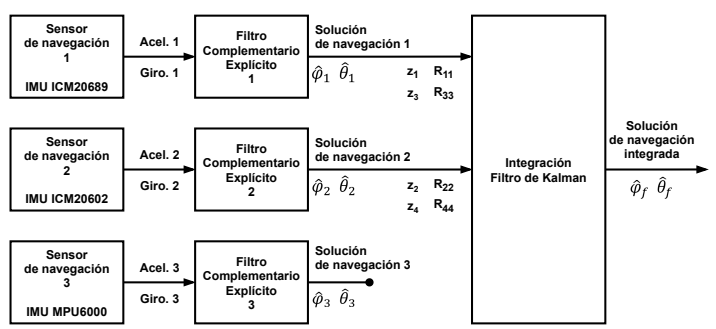

Figura 4: Arquitectura de integración filtrada en cascada de estado total.

Es importante mencionar que los errores presentes en los sistemas de navegación comprenden una mezcla de errores sistemáticos y ruido blanco, los cuales deben modelarse de forma correcta, que se suavizarán a través del KF.

Debe comentarse que, al igual que sucedía con el subsistema tomado como referencia, esta estructura tampoco plantea realimentación, la cual reduce la independencia entre los subsistemas dificultando la detección de problemas en los sensores, al no poder comparar directamente los resultados. Por tanto, la ausencia de esta característica permite identificar de forma directa posibles mal funcionamientos de los sensores implicados.

En la tabla 2 se muestran los parámetros tras el proceso de tuning para los controladores PI que intervienen en los ECF.

Tabla 2: Parámetros de los controladores PI.

\begin{tabular}{|l|l|l|}
\hline Sensor & kp & ki \\
\hline ICM20689 & 1.2000 & 0.014 \\
ICM20602 & 0.0001 & 0.300 \\
MPU6000 & 0.0001 & 0.050 \\
\hline
\end{tabular}

\subsection{ESTRATEGIA 2: INTEGRACIÓN FILTRADA CENTRALIZADA DE ESTADO TOTAL MODIFICADA}

En este caso se utiliza una modificación de la arquitectura de integración filtrada centralizada de estado total (Figura 5).

De forma general, un filtro de estado total es adecuado para integrar sistemas de determinación de la posición. 
Por su parte, un filtro de estado de error, más complejo al trabajar en lazo cerrado y en lazo abierto de forma simultánea, se utiliza en sistemas que disponen de sensores navegación inercial.

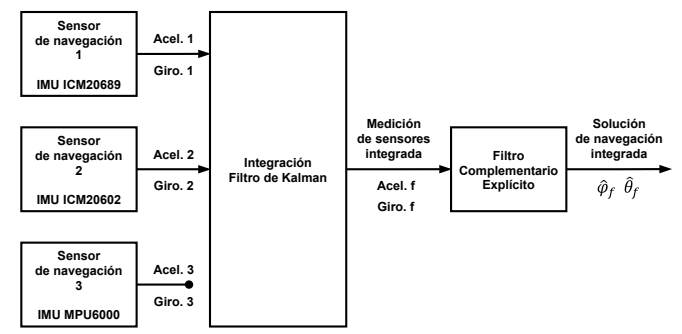

Figura 5: Arquitectura modificada de integración filtrada centralizada de estado total.

En este trabajo, a pesar de emplear sensores inerciales, se parte de un filtro de estado total al cual se le aplica una modificación: desacoplar un KF complejo, dependiendo de las características dinámicas del sistema puede ser Extendido o Unscented, en un filtro de Kalman lineal y un filtro complementario explícito. Dicho cambio simplifica los filtros, los cuales siguen siendo válidos para el sistema, facilita el ajuste de sus parámetros y reduce el coste computacional, bastante elevado en este tipo de arquitectura. Esto también es posible gracias a que las mediciones de una IMU no incorporan de forma predeterminada ningún algoritmo de estimación, siendo adecuadas para una arquitectura centralizada.

A diferencia de la arquitectura en cascada, las medidas de los sensores se integran directamente en un filtro de Kalman, es decir, los datos raw de los acelerómetros y de los giroscopios en lugar de las estimaciones. Al igual que en la situación anterior, para este proceso de fusión solo se emplean dos de las IMUs. De esta forma se obtienen datos de acelerómetro y giroscopio filtrados y compensados, gracias a las características dinámicas de los sensores, que se pasan a un ECF, tal y como se ha adelantado anteriormente, para estimar los valores definitivos de roll y de pitch.

En esta arquitectura, al disponer de un único filtro de Kalman, los errores sistemáticos y el ruido de todos los sensores se modelan en el mismo algoritmo. Esto asegura la correlación entre errores y la ponderación de las mediciones, pero requiere un diseño exhaustivo del filtro por la gran cantidad de información a definir. Además, como ya se ha comentado anteriormente, su principal desventaja es el elevado coste computacional que acarrea, el cual se reduce gracias a la modificación efectuada.

Para este caso, los parámetros del controlador para el ECF son ki $=0.250$ y $\mathrm{kp}=0.014$.

\section{RESULTADOS}

Para la consecución de los resultados presentados se realizan pruebas introduciendo en las IMUs una velocidad angular para cada eje, de tal forma que se genera una rotación constante que cubre un amplio rango en el que puede funcionar un sensor de este tipo. En cuanto a la aceleración lineal, se aporta una señal aleatoria que actúa como ruido del sistema, simulando, de forma simplificada, las aceleraciones que pueden afectar a un UAV.

En la Figura 6 se muestran los valores de roll $\left(^{\circ}\right)$ y de pitch $\left(^{\circ}\right)$ obtenidos a partir de los ECF que se implementan de forma directa sobre cada IMU en la primera estrategia. Asimismo, estos resultados se comparan con el valor de referencia, calculado a partir de la velocidad angular aplicada al sistema.
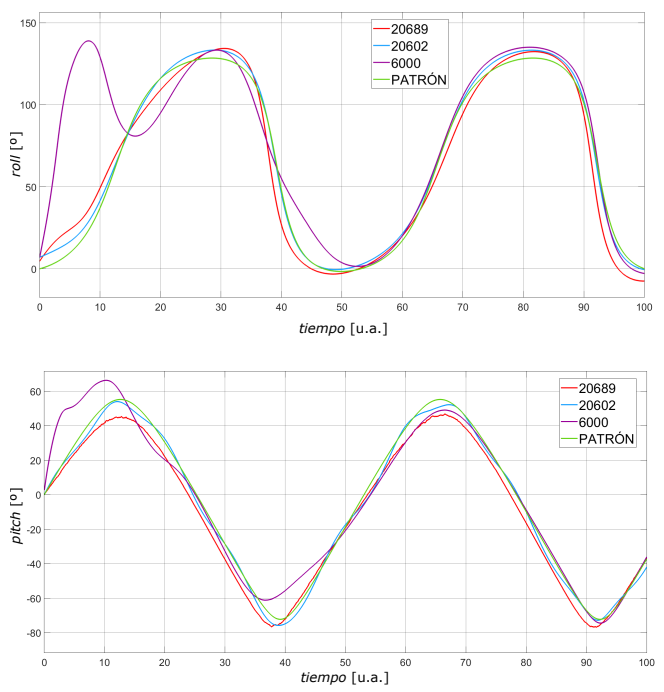

Figura 6: Estimación de roll (arriba) y pitch (abajo) para cada una de las IMUs.

Ambas gráficas obedecen a un comportamiento similar: el sensor ICM20602 es el que más se ajusta al valor patrón, mientras que, tal y como se esperaba, el MPU6000 se aleja de forma clara de la referencia al comienzo del experimento debido a su bias, aunque mejora su estimación a lo largo del tiempo debido a la estabilización del filtro.

En la Figura 7 se presenta el resultado de fusionar en la segunda estrategia los datos de los acelerómetros $\left(\mathrm{m} / \mathrm{s}^{2}\right)$, en sus tres componentes, correspondientes a ICM20689 e ICM20602. Se puede observar cómo mediante el KF se logra reducir el ruido de las señales originales y compensar las características dinámicas de cada sensor.

Una vez expuestos los datos intermedios que se obtienen en los dos casos planteados, a continuación, se muestran las estimaciones de roll y de pitch $\left(^{\circ}\right)$ para cada una de las estrategias (Figura 8), mostrando también la referencia. 


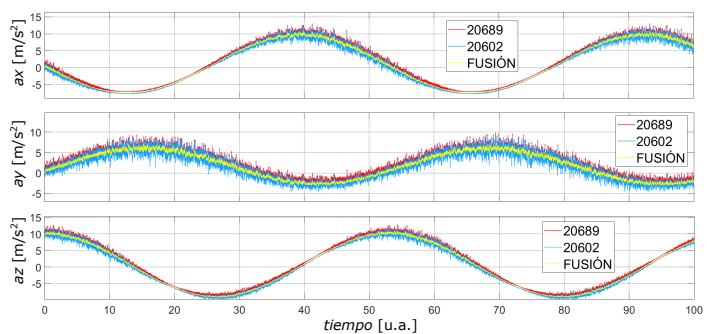

Figura 7: Fusión de acelerómetros en la segunda estrategia.

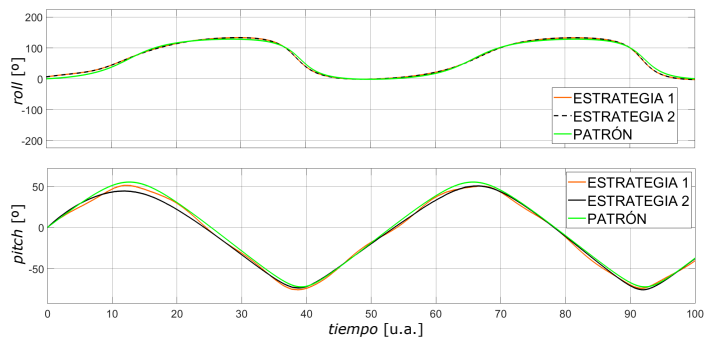

Figura 8: Estimaciones de roll y pitch en cada estrategia.

Se aprecia que ambas estructuras estiman resultados muy similares, por lo que la integración de los valores estimados de forma individual para el primer caso o de los datos raw de los sensores en el segundo no tiene influencia relevante en los valores finales de actitud.

Para poder comparar de forma cuantitativa los resultados y confirmar el análisis previo, se exhiben los errores angulares $\left(^{\circ}\right)$ dinámicos para roll y para pitch (Figura 9), mostrando en las sub-gráficas superiores el error de las estimaciones individuales de los sensores y en las inferiores el error tras los procesos de integración.
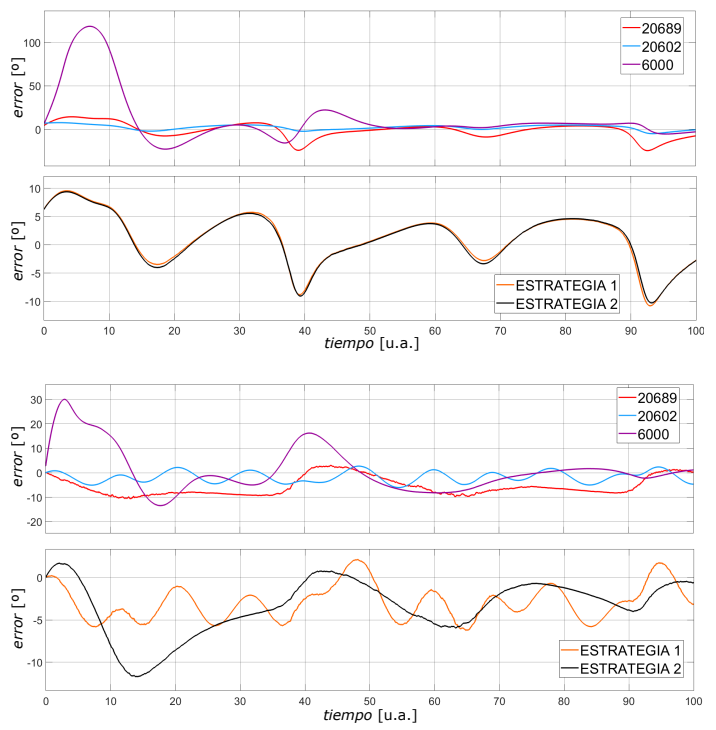

Figura 9: Errores angulares dinámicos en roll (arriba) y en pitch (abajo).
En lo que a roll se refiere, se advierte que el error de ICM20689 oscila entre $+15^{\circ}$ y $-25^{\circ}$. En estas gráficas se corrobora que ICM20602 es la IMU que mejor comportamiento presenta, al tener como valores más alejados de la referencia $+8^{\circ} \mathrm{y}-4^{\circ}$, consiguiendo una estimación bastante precisa. En cuanto a MPU6000, debido a su gran bias presenta un error elevado al comienzo del experimento, aunque mejora progresivamente manteniéndose entre $\pm 20^{\circ}$. Por otro lado, tal y como se ha comentado, las dos estrategias de integración arrojan resultados muy similares, encontrándose sus errores entre $\pm 10^{\circ}$ en ambos casos.

Algo similar sucede al representar el error angular de pitch. De nuevo, el sensor que presenta un mejor comportamiento es ICM20602, aunque en los tres casos se reduce el error. Sin embargo, la primera estrategia de fusión muestra mejores resultados puesto que su error varía entre $+2^{\circ}$ y $-6^{\circ}$, frente $\mathrm{a}+2^{\circ} \mathrm{y}-12^{\circ}$ de la segunda.

Para completar la comparación de todas las estimaciones de actitud ejecutadas, en la Figura 10 se presentan los errores RMS asociados a las simulaciones para cada una de las IMUs.

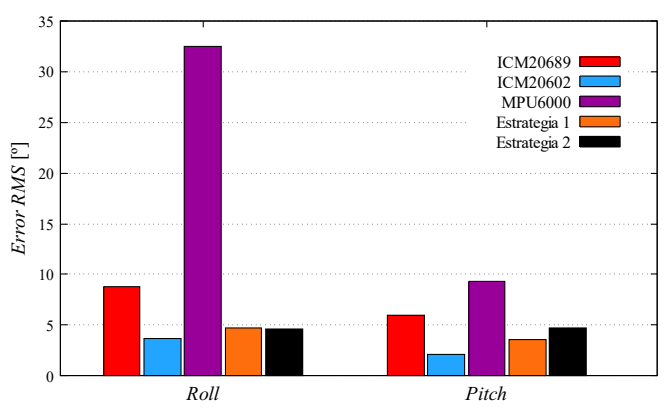

Figura 10: Error RMS en las estimaciones.

Para ilustrar la carga computacional referente a cada estrategia, se realizan varias simulaciones, con una duración de 100 unidades arbitrarias en Simulink, de las distintas opciones para estimar la actitud. A continuación, se recogen los tiempos medios de ejecución:

- ICM20602: 50.48 segundos

- Estrategia 1: 74.63 segundos

- Estrategia 2: 57.32 segundos

A raíz de los resultados obtenidos para las dos estrategias, se confirma que el desacoplo de un filtro de Kalman, que agrupa todos los parámetros, en otro más sencillo combinado con un ECF (estrategia 2) supone un tiempo de ejecución inferior al arrojado por la estrategia 1, en la cual se implementan tres ECF en paralelo y posteriormente un KF, implicando un mayor coste computacional para el microcontrolador. 


\section{CONCLUSIONES}

En base a los resultados presentados y tras el análisis de los mismos, se puede afirmar que las mejoras obtenidas en las dos estrategias de integración frente a los resultados del sensor que menor error presenta, ICM20602, no son suficientes para justificar el coste computacional que implica la ejecución de los filtros para cada estrategia. Aunque se reduce el ruido de las medidas, especialmente al emplear el KF con los datos raw del acelerómetro y del giroscopio, los resultados de actitud alcanzados mediante fusión no reducen el error respecto a los valores de referencia.

Por estos motivos, se plantea como solución final para la estimación de actitud según las particularidades de nuestro sistema una arquitectura en redundancia, independiente para roll y pitch, que dispone de tres IMUs trabajando de forma simultánea: una de ellas se toma como referencia para calcular el valor de actitud que recibe el sistema de control, aunque las otras dos están en funcionamiento en todo momento. Esta estructura permite solucionar de forma casi inmediata el fallo de alguna de ellas sin perder la información relativa a la actitud del cuerpo.

El dispositivo predeterminado es ICM20602 debido a sus prestaciones (ver Figuras 9 y 10). Esta será la IMU principal mientras su error angular instantáneo en valor absoluto para roll y pitch sea inferior a $10^{\circ}$ y a $5^{\circ}$, respectivamente. Si se supera dicho umbral, automáticamente se toma como sensor de referencia ICM20689 que será el encargado de proporcionar los datos mientras ICM20602 no recupere la normalidad o cuando presente errores angulares inferiores a $25^{\circ}$ y $15^{\circ}$ para los ángulos en cuestión. En caso contrario, y como último recurso, se toman los datos extraídos de MPU6000 para implementar el sistema de control.

Tal y como se observa en la Figura 6, dado que la actitud estimada por MPU6000 difiere en gran medida del patrón al inicio del experimento, la monitorización de la IMU de referencia puede servir para informar al piloto del estado del vehículo. De acuerdo a la estructura de redundancia planteada, una activación temprana de dicha IMU no solo evidenciaría un malfuncionamiento de los otros dos sensores sino que también supondría un riesgo para el control del UAV.

Para terminar de ilustrar el funcionamiento de este sistema, en la Figura 11 se muestra la estimación de pitch. En la prueba mostrada se altera el comportamiento normal de ICM20602 para que entre en funcionamiento ICM20689 y poder hacer frente a las anomalías detectadas, tal y como se observa en las zonas remarcadas de la gráfica.

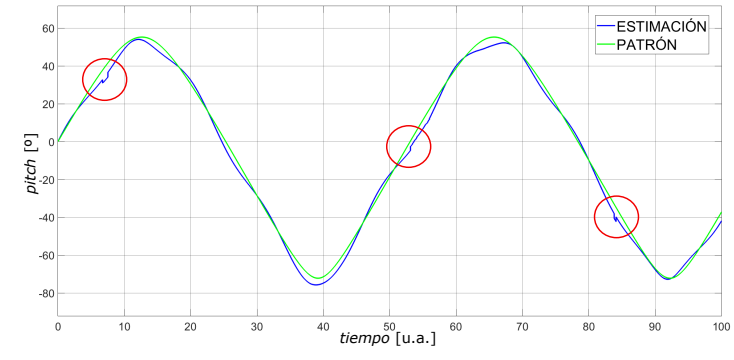

Figura 11: Funcionamiento de la estructura en redundancia para pitch.

\section{Agradecimientos}

Los autores agradecen la ayuda prestada por el Gobierno de La Rioja a través del proyecto de $\mathrm{I}+\mathrm{D}$ ADER 2017-I-IDD-00035 y por la Universidad de La Rioja a través de la Ayuda a Grupos de Investigación REGI 2020/23.

\section{English summary}

\section{COMPARISON OF STRATEGIES FOR ATTITUDE ESTIMATION IN FLIGHT CONTROLLER FOR PROFESSIONAL UAV}

\begin{abstract}
This work presents two multisensor integration strategies by which is intended to find an appropriate method for estimating the attitude of a drone using Kalman filters and explicit complementary filters. For this purpose, low-cost sensors are simulated, specifically, the Inertial Measurement Units, which are incorporated in a flight controller designed for a professional Unmanned Aerial Vehicle. In this way, reliable data is available and it can be of great interest to address the start up of the system in real tests, allowing for implementation its control system properly based on this information. This application is developed in MATLAB-Simulinkß, as it is a suitable environment for working with $d y$ namic systems.
\end{abstract}

Keywords: Attitude Estimation, Inertial Measurement Unit, Explicit Complementary Filter, Kalman Filter, Flight Controller, Professional UAV. 


\section{Referencias}

[1] Belter, D., \& Nowicki, M. R. (2019). Optimization-based legged odometry and sensor fusion for legged robot continuous localization. Robotics and Autonomous Systems, 111, 110-124.

[2] Chérigo, C., \& Rodríguez, H. (2017). Evaluación de algoritmos de fusión de datos para estimación de la orientación de vehículos aéreos no tripulados. RIDTEC, 13(2), 90-99.

[3] Euston M, Coote P, Mahony R, Kim J, Hamel T. A Complementary Filter for Attitude Estimation of a Fixed-Wing UAV. IEEE/RSJ International Conference on Intelligent Robots and Systems, IROS. 2008;340-5.

[4] Fu, C., Hong, W., Lu, H., Zhang, L., Guo, X., \& Tian, Y. (2018). Adaptive robust backstepping attitude control for a multi-rotor unmanned aerial vehicle with time-varying output constraints. Aerospace Science and Technology, 78, 593-603.

[5] Fung, M. L., Chen, M. Z. Q., \& Chen, Y. H. (2017). Sensor fusion: A review of methods and applications. Proceedings of the 29th Chinese Control and Decision Conference, CCDC 2017, 3853-3860.

[6] García, J., Molina, J. M., \& Trincado, J. (2020). Real evaluation for designing sensor fusion in UAV platforms. Information Fusion, $63,136-152$.

[7] Groves, P. D. (2013). Principles of GNSS, Inertial, and Multisensor Integrated Navigation Systems (2nd ed.). Artech House.

[8] Hoang, M. L., \& Pietrosanto, A. (2021). Yaw/Heading optimization by drift elimination on MEMS gyroscope. Sensors and Actuators, A: Physical, 325, 112691.

[9] Jin, R., Jiang, J., Qi, Y., Lin, D., \& Song, T. (2019). Drone Detection and Pose Estimation Using Relational Graph Networks. Sensors, 19(1476).

[10] Khaleghi, B., Khamis, A., Karray, F. O., \& Razavi, S. N. (2013). Multisensor data fusion: A review of the state-of-the-art. Information Fusion, 14(1), 28-44.

[11] Kim, P. (2011). Kalman Filter for Beginners: with MATLAB Examples.

[12] Kim, T., \& Park, T. H. (2020). Extended kalman filter (Ekf) design for vehicle position tracking using reliability function of radar and lidar. Sensors (Switzerland), 20(15), $1-18$.
[13] Li, S., Ozo, M. M. O. I., De Wagter, C., \& de Croon, G. C. H. E. (2020). Autonomous drone race: A computationally efficient visionbased navigation and control strategy. Robotics and Autonomous Systems, 133.

[14] Ma, H., Yan, L., Xia, Y., \& Fu, M. (2020). Kalman Filtering and Information Fusion. Springer.

[15] Mahony, R., Hamel, T., \& Pflimlin, J.M. (2008). Non-linear complementary filters on the special orthogonal group. IEEE Transactions on Automatic Control, 53(5), 1203-1218.

[16] Noordin, A., Basri, M. A. M., \& Mohamed, Z. (2018). Sensor Fusion Algorithm by Complementary Filter for Attitude Estimation of Quadrotor with Low-cost IMU. Telkomnika, $16(2), 868-875$.

[17] Rico-Azagra, J., Gil-Martínez, M., RicoAzagra, R., \& Maisterra, P. (2016). Low-cost attitude estimation for a ground vehicle. Advances in Intelligent Systems and Computing, 417, 121-132.

[18] Rico-Azagra J, Rico R, Maisterra P, GilMartínez M. Comparación de algoritmos de estimación de actitud. En: XXXVI Jornadas de Automática. Bilbao, España: CEA-IFAC; 2015. p. 911-20.

[19] Svacha, J., Mohta, K., Watterson, M., Loianno, G., \& Kumar, V. (2018). Inertial Velocity and Attitude Estimation for Quadrotors. IEEE International Conference on Intelligent Robots and Systems, 7810-7816.

[20] Verma, M., Dehaeze, T., Zhao, G., Watchi, J., \& Collette, C. (2021). Virtual sensor fusion for high precision control. Mechanical Systems and Signal Processing, 150.

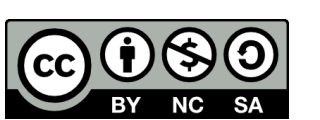

C) 2021 by the authors. Submitted for possible open access publication under the terms and conditions of the Creative Commons Attribution CC BY-NC-SA 4.0 license (https://creativecommons.org/licenses/by-ncsa/4.0/deed.es). 